\title{
Koenigs-Knorr Glycosylation with Neuraminic Acid Derivatives
}

\author{
Galina Pazynina, ${ }^{1}$ Vitaly Nasonov, ${ }^{1}$ Ivan Belyanchikov, ${ }^{1}$ Reinchard Brossmer, ${ }^{2}$ \\ Maxim Maisel, ${ }^{1}$ Alexander Tuzikov, ${ }^{1}$ and Nicolai Bovin ${ }^{1}$ \\ ${ }^{1}$ Shemyakin and Ovchinnikov Institute of Bioorganic Chemistry, Russian Academy of Sciences, ul. Miklukho-Maklaya 16/10, \\ Moscow 117997, Russia \\ ${ }^{2}$ Biochemistry Center Heidelberg, University of Heidelberg, 69120 Heidelberg, Germany
}

Correspondence should be addressed to Nicolai Bovin, bovin@ibch.ru

Received 13 July 2010; Revised 15 September 2010; Accepted 21 October 2010

Academic Editor: Richard D. Cummings

Copyright (C) 2010 Galina Pazynina et al. This is an open access article distributed under the Creative Commons Attribution License, which permits unrestricted use, distribution, and reproduction in any medium, provided the original work is properly cited.

\begin{abstract}
Earlier we reported a convenient and efficient method of preparing $\alpha 2-6$ sialooligosaccharides in conditions of Koenigs-Knorr reaction. The use of $\mathrm{Ag}_{2} \mathrm{CO}_{3}$ allowed carrying out $\alpha$ 2-6 sialylation of galacto-4,6-diol of mono- and disaccharides with chloride of acetylated $\mathrm{N}$-acetylneuraminic acid methyl ester as glycosyl donor. In this study we applied this approach to other derivatives of neuraminic acid, namely, Neu5Gc, 9-deoxy-9-NAc-Neu5Ac, Neu5Ac $\alpha 2-8$ Neu5Ac, and Neu5Ac $\alpha 2-8$ Neu5Ac $\alpha 2-8$ Neu5Ac as glycosyl donors; eight compounds were synthesized: Neu5Gc $\alpha-\mathrm{O}\left(\mathrm{CH}_{2}\right)_{3} \mathrm{NH}_{2}(\mathbf{8}), \mathrm{Neu} 5 \mathrm{Gc} \alpha 2-6 \mathrm{Gal} \beta 1-4 \mathrm{GlcNAc} \beta-\mathrm{O}\left(\mathrm{CH}_{2}\right)_{3} \mathrm{NH}_{2}$ (10), 9-deoxy-9-NAc-Neu5Ac-O $\left(\mathrm{CH}_{2}\right)_{3} \mathrm{NH}_{2}$ (15), 9-deoxy-9-NAc-Neu5Ac $\alpha 2-6 \mathrm{Gal} \beta 1-4 \mathrm{GlcNAc} \beta-\mathrm{O}\left(\mathrm{CH}_{2}\right)_{3} \mathrm{NH}_{2}(\mathbf{1 7}), \mathrm{Neu}^{\mathrm{Ac}} \alpha 2$ $8 \mathrm{Neu} 5 \mathrm{Ac} \alpha-\mathrm{O}\left(\mathrm{CH}_{2}\right)_{3} \mathrm{NH}_{2}(23) \mathrm{Neu} 5 \mathrm{Ac} \alpha 2-8 \mathrm{Neu} 5 \mathrm{Ac} \alpha-\mathrm{OCH}_{3}$ (24), Neu5Ac $\alpha 2-8 \mathrm{Neu} 5 \mathrm{Ac} \alpha-\mathrm{OCH}_{2}\left(p-\mathrm{C}_{6} \mathrm{H}_{4}\right) \mathrm{NHCOCH}_{2} \mathrm{NH}_{2}(\mathbf{2 5})$, and Neu5Ac $\alpha 2-8 \mathrm{Neu} 5 \mathrm{Ac} \alpha 2-8 \mathrm{Neu} 5 \mathrm{Ac} \alpha-\mathrm{O}\left(\mathrm{CH}_{2}\right)_{3} \mathrm{NH}_{2}$ (32). These sialosides were used for characterization of siglecs and other carbohydrate-binding proteins.
\end{abstract}

\section{Introduction}

Sialic acid family comprises over 40 neuraminic acid versions and monosaccharide derivatives; the number of biologically relevant sialic acid oligosaccharides is also very high. Sialic acids are a part of the most important molecules of life, since they occupy the terminal position on cell membrane glycoproteins and glycolipids. Given their location and ubiquitous distribution, sialic acids can mediate or modulate a wide range of physiological and pathological processes and play a role not only in the protection and adaptation of life, but also in being utilized by life-threatening infectious microorganisms [1]. Several families of sialic acidbinding proteins have been discovered over the last few decades, including mammalian selectins and siglecs [2]. Only a limited number of sialomolecules are available as the tools for the study of mammalian and microbial lectins and sialo-modifying enzymes. Due to this, the search of simple and efficient synthetic ways for sialosaccharides is very relevant. The methods of sialylation described in the literature [3-5] rarely give high yields and $\alpha$-stereoselectivity but suggest complex modifications of donors; introduction and removal of substituents at C-3, C-4, C-5, and C-9 in order to improve sialylation stereoselectivity presume complex multistage synthesis of such compounds. In case of the use of 2-xantates, thiophosphates and various imidates, sialylation usually proceeds at $-50-70^{\circ} \mathrm{C}$ and requires strong Lewis acids as promoters, this posing the corresponding limitations to the repertoire of protective groups in glycosyl acceptor. Recently we have described a simple and efficient method of the synthesis of $\alpha-2-6$ sialooligosaccharides in conditions of Koenigs-Knorr reaction [6] when the simplest possible sialyl donor is used at room temperature ang gives high $\alpha$-stereoselectivity. It was interesting to study the applicability of this approach to other neuraminic acid derivatives (Neu5Gc, 9-deoxy-9-NAc-Neu5Ac, Neu5Ac $\alpha 2$ $8 \mathrm{Neu} 5 \mathrm{Ac}$, and Neu5Ac $\alpha 2-8 \mathrm{Neu} 5 \mathrm{Ac} \alpha 2-8 \mathrm{Neu} 5 \mathrm{Ac}$ ). Here, we describe practical chemical syntheses of several spacerarmed sialic acid glycosides, with especial interest to $\mathrm{N}$ glycolyl-neuraminic acid version playing role in cancer 
immunology and transplantation, and also $\alpha 2-8$ oligosaccharides known as key motif of polysialic acids, numerous gangliosides, and serving as affinity ligand for siglec-7 [7].

Peracetylated methyl esters of Neu5Gc (6) [8], 9-deoxy9-NAc-Neu5Ac (13), Neu5Ac $\alpha 2-8 \mathrm{Neu} 5 \mathrm{Ac}$ (19 and 20) [9], and Neu5Ac $\alpha 2-8$ Neu5Ac $\alpha 2-8$ Neu5Ac (27-30) [10] were synthesized starting from free saccharides by esterification in acidic conditions $(\mathrm{MeOH} / \mathrm{HCl})$ with subsequent acetylation $\left(\mathrm{Ac}_{2} \mathrm{O} /\right.$ pyridine).

2-Chloroderivatives 7, 14, $21+22$, and 31, Sug-Cl, were synthesized from the corresponding peracetylated methyl esters by treatment with $\mathrm{HCl}$ in $\mathrm{CHCl}_{3}$ for $1.5-2$ days and were used without further purification.

Glycosylation was performed in dry $\mathrm{CH}_{2} \mathrm{Cl}_{2}$ in the presence of $\mathrm{Ag}_{2} \mathrm{CO}_{3}$ or $\mathrm{Ag}_{2} \mathrm{CO}_{3} / \mathrm{AgOTf}$ (silver trifluoromethanesulfonate) and molecular sieves $4 \AA$ (MS $4 \AA$ ) at room temperature for 1-7 days; the reaction conditions and yields are given in the Table 1. In this study we have used equivalent amounts of glycosyl donor (Sug-Cl) and acceptor or excess of acceptor. The corresponding glycals were found to be the main side products in all glycosylation reactions. Deprotection resulted in the mixture of the target product with glycal and unreacted acceptor, easily separated using cation-exchange chromatography on Dowex $\mathrm{H}^{+}$resin [11]: elution with water gave glycal (acid), whereas the product (amino acid) had been retained on the column and was completely eluted with $1 \mathrm{M}$ aqueous pyridine; unreacted glycosyl acceptor (amine) was eluted with $1 \mathrm{M}$ $\mathrm{NH}_{4} \mathrm{OH}$. In the case of disialic and trisialic glycosides partial lactonization occurred during purification on Dowex $\mathrm{H}^{+}$, so the lactone was treated with aqueous $\mathrm{NaOH}$ to give corresponding acid.

All Sug-Cl (see Table 1) have demonstrated considerably less activity in glycosylation compared to chloride of acetylated $N$-acetylneuraminic acid methyl ester $(3)[6,8]$; in several cases $\mathrm{Ag}_{2} \mathrm{CO}_{3}$ promoted glycosylation (without AgOTf) either proceeded very slowly or did not proceed at all. The optimal of AgOTf added to $\mathrm{Ag}_{2} \mathrm{CO}_{3}$ ratio (in respect to the reaction duration and acceptable $\alpha / \beta$ anomer ratio) was found to be $10 \%$ mol using chloride of Neu5Ac (3) as glycosyl donor and $\mathrm{HO}\left(\mathrm{CH}_{2}\right)_{3} \mathrm{NHCOCF}_{3}$ as acceptor; larger amount gave more $\beta$-isomer and glycal; preferable acceptor/donor ratio was not less than 2. Glycosylation at $-10^{\circ} \mathrm{C}$ did not improve the $\alpha / \beta$ ratio compared to room temperature.

Glycosylation with chloride of Neu5Gc (7) catalyzed by $\mathrm{Ag}_{2} \mathrm{CO}_{3}$ in absence of AgOTf proceeded slowly; 3trifluoroacetamidopropanol (Scheme 1, no. 3 in Table 1) gave the mixture of anomers in $68 \%$ yield $(\alpha / \beta$ 92:8). Deprotected product was isolated on Dowex $\mathrm{H}^{+}$resin (elution with $1 \mathrm{M}$ Py) with additional HPLC purification; the corresponding $\alpha$-glycoside (8) was isolated in $57 \%$ yield, that was better result compared to NIS/TFS-OH promoted glycosylation with Neu5Gc $\beta$ SEt [8].

Glycosylation of $4^{\prime}, 6^{\prime}$-diol of $N$-acetyllactosamine (9) with chloride of Neu5Gc 7 in presence of $\mathrm{Ag}_{2} \mathrm{CO}_{3} / \mathrm{AgOTf}$ (Scheme 1, no. 4 in Table 1 ) gave $30 \%$ of $\alpha / \beta$ mixture (43:57); the pure $\alpha$-glycoside 10 was isolated (HPLC) in
$12 \%$ yield. Unreacted acceptor 9 (58\%) was recovered in deprotected form 11.

Glycosylation with chloride of 9-deoxy-9-NAc-Neu5Ac 14 in presence of only $\mathrm{Ag}_{2} \mathrm{CO}_{3}$ proceeded similarly to the reaction with Neu5Ac (Scheme 2, no. 5 and no. 6 in Table 1). 3-Trifluoroacetamidopropanol and 4',6'-diol 16 gave comparable yields of corresponding $\alpha$-glycosides (29\% of 15 and $25 \%$ of 17); $\beta$ anomers were observed in trace amounts. Unreacted acceptor 16 was recovered $(66 \%)$ in deprotected form 11.

Methylation followed by acetylation of Neu5Ac $\alpha 2-$ $8 \mathrm{Neu} 5 \mathrm{Ac}$ (18) or Neu5Ac $\alpha 2-8 \mathrm{Neu} 5 \mathrm{Ac} \alpha 2-8 \mathrm{Neu} 5 \mathrm{Ac}$ (26) gave the mixtures of acetates of methyl esters and corresponding lactones (preparation of the lactones described in $[9,10])$; the mixtures were used without separation. Acetates of disialic acid were the mixture of 19 and 20 (yield 82\%); 2Chloroderivatives used as glycosyl donor were the mixture of 21 and 22 (Scheme 3). Acetates of trisialic acid were the mixture of four substances (27-30) (yield 72\%); the mixture of corresponding four 2-Chloroderivatives used as glycosyl donor is designated as 31 (Scheme 4).

Glycosylation with Neu5Ac $\alpha 2-8 \mathrm{Neu} 5 \mathrm{Ac}$ glycosyl chlorides $21+22$ in absence of AgOTf proceeded very slowly. Spacer alcohol $\mathrm{HO}\left(\mathrm{CH}_{2}\right)_{3} \mathrm{NHCOCF}_{3}$ gave $\sim 20 \%$ yield of glycosylation product $23, \alpha / \beta$ ratio $94: 6$. Glycosylation in the presence of AgOTf proceeded seven times faster resulting in $\alpha / \beta$ ratio $90: 10$ (32\%) (Scheme 3, no. 7 and no. 8 in Table 1). Synthesis of disialic acid methyl glycoside was carried out at 100-fold excess of methanol (Scheme 3, no. 9 in Table 1). After deprotection and chromatography on silica gel, purification on Sephadex LH-20 and ion exchange to $\mathrm{Na}^{+}$on Dowex the yield of target $\alpha$-glycoside 24 was $40 \%$ ( $\sim 33 \%$ on starting disialic acid) that is comparable to the literature data [12]; only the traces of $\beta$ glycoside were observed.

In case of $p$-amidoglycyl-benzyl alcohol glycosylation with Neu5Ac $\alpha 2-8 \mathrm{Neu} 5 \mathrm{Ac}$ the use of $0.1 \mathrm{~mol}$ of AgOTf per glycosyl chloride gave only $10 \%$ of $25 ; 0.6$ ratio led to increase of conversion (yield of $\alpha+\beta$ increased from $20 \%$ to $32 \%$ ), but it was accompanied with the increase of $\beta$-anomer formation (Scheme 3, no. 10 and no. 11 in Table 1).

Trisialic chlorides 31 reacted with $\mathrm{HO}\left(\mathrm{CH}_{2}\right)_{3} \mathrm{NHCOCF}_{3}$ only in the presence of AgOTf; increase of $\mathrm{mol} / \mathrm{mol}$ ratios from 0.1 to 0.5 on chloride gave 32 ( $\sim 25 \%$ yield) accompanied by the increase of conversion degree and reduction of $\alpha / \beta$ ratio (Scheme 4 , no. 12 and no. 13 in Table 1 ).

The structure of all the studied compounds was confirmed by high resolution ${ }^{1} \mathrm{H}$ NMR data. Spectra of derivatives $4,8,10$, and 24 coincided with those described before $[8,12,13]$. Chemical shift of $\mathrm{H}-3$ eq (Neu5Ac, Neu5Gc and 9-deoxy-9-NAc-Neu5Ac residues) was 2.6$2.7 \mathrm{ppm}$ for $\alpha$-anomer and 2.2-2.4 ppm for $\beta$-anomer [3]. Upfield shift has been observed in spectra of 9-deoxy-9-NAcNeu5Ac derivatives for $\mathrm{H}-9$ (3.6 ppm) and $\mathrm{H}^{\prime}$ (3.3 ppm) as compared to Neu5Ac (3.9 and $3.6 \mathrm{ppm}$, correspondingly) [14]. Glycosidic $\alpha(2-8)$ bond in disialic and trisialic acid derivatives was characterized by downfield position of $\mathrm{H}$ 8 and H-9 (>4.0 ppm) as compared to Neu5Ac [12, 15]. The synthesized sialosides were used for characterization of 


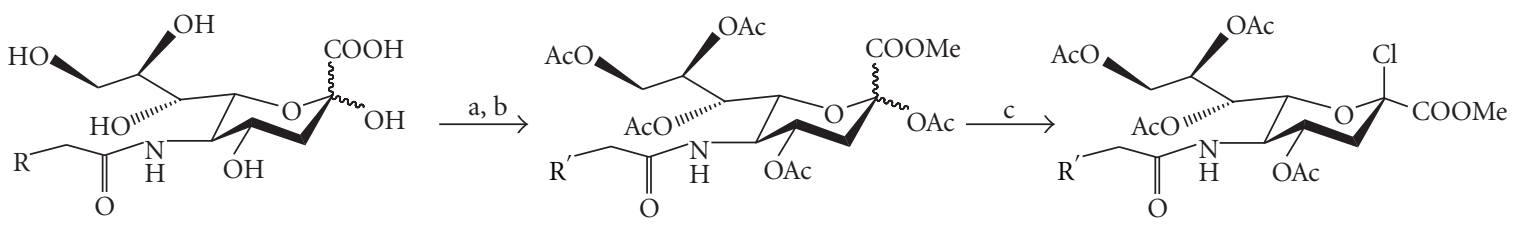
$1 \mathrm{R}=\mathrm{H}$
$2 \mathrm{R}^{\prime}=\mathrm{H}$
$3 \mathrm{R}^{\prime}=\mathrm{H}$
$5 \mathrm{R}=\mathrm{OH}$
$6 \mathrm{R}^{\prime}=\mathrm{OAC}$
$7 \mathrm{R}^{\prime}=\mathrm{OAC}$

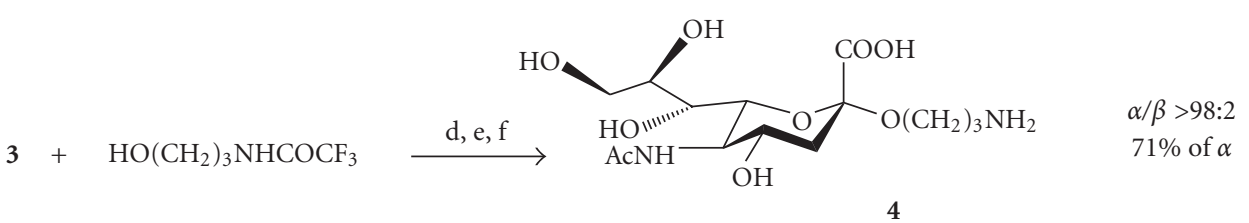

$7+\mathrm{HO}\left(\mathrm{CH}_{2}\right)_{3} \mathrm{NHCOCF}_{3} \stackrel{\mathrm{d}, \mathrm{e}, \mathrm{f}}{\longrightarrow}$

$7+$

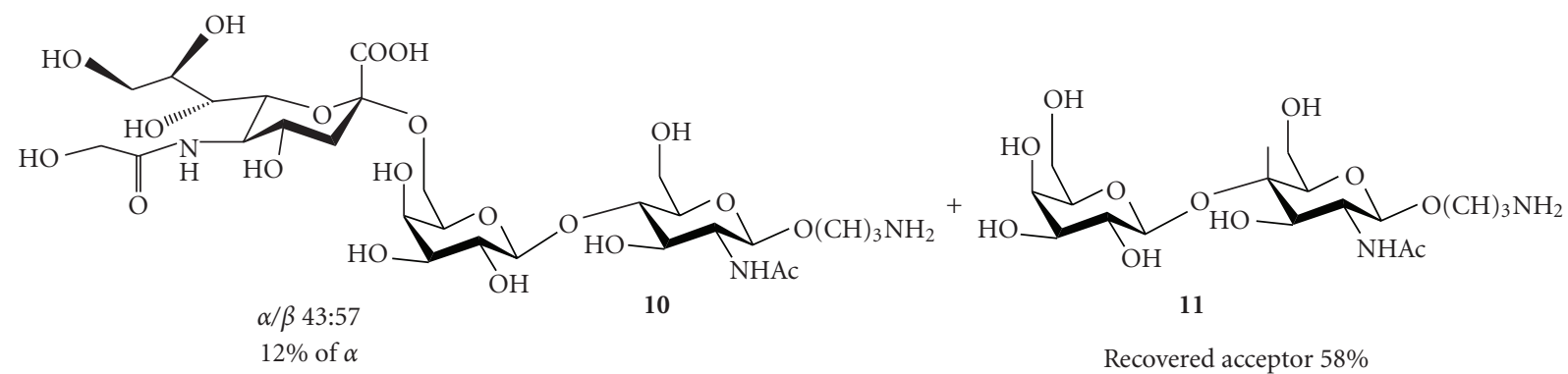

Scheme 1: (a) MeOH/HCl; (b) $\mathrm{Ac}_{2} \mathrm{O} / \mathrm{Py}$; (c) $\mathrm{AcCl}, \mathrm{MeOH} / \mathrm{CHCl}_{3}$; (d) $\mathrm{Ag}_{2} \mathrm{CO}_{3}\left(\mathrm{Ag}_{2} \mathrm{CO}_{3} / \mathrm{AgOTf}\right.$ ), $\mathrm{CH}_{2} \mathrm{Cl}_{2}, \mathrm{MS} 4 \AA$; (e) $\mathrm{MeONa} / \mathrm{MeOH}$; (f) $\mathrm{NaOH} / \mathrm{H}_{2} \mathrm{O}$; (g) $\mathrm{H}_{2}, \mathrm{Pd} / \mathrm{C}$.

siglecs [16] and other carbohydrate-binding proteins [17, $18]$.

\section{Experimental}

Neu5Ac was from Juelich Enzyme Products GmbH (Wiesbaden, Germany); 9-deoxy-9-NAc-Neu5Ac was obtained as described in [19]; Neu5Ac $\alpha 2-8$ Neu5Ac and Neu5Ac $\alpha 2-$ $8 \mathrm{Neu} 5 \mathrm{Ac} \alpha 2-8 \mathrm{Neu} 5 \mathrm{Ac}$ and Neu5Gc were from Nacalai Tesque Inc. (Kyoto, Japan).

2.1. Preparation of Peracetylated Methyl Esters of Neuraminic Acid Derivatives General procedure for 6, 13, $19+20$, and
27-30. Neuraminic acid derivative $(0.5 \mathrm{mmol}$ of $5, \mathbf{1 2}, \mathbf{1 8}$, or 26 as $\mathrm{Na}$ salt) was suspended in $25 \mathrm{ml}$ of dry methanol cooled to $0^{\circ} \mathrm{C}$ followed by dropwise addition of $\mathrm{AcCl}(178 \mu \mathrm{l}$, $2.5 \mathrm{mmol}$ ). The mixture was stirred at room temperature for $2 \mathrm{~h}$ (clear solution is formed). The solution was evaporated at reduced pressure (water jet pump) and coevaporated three times with toluene. The residue was acetylated with $9 \mathrm{ml}$ of $\mathrm{Ac}_{2} \mathrm{O} / \mathrm{Py}(1: 2)$ for $15-20 \mathrm{~h}$ at $40^{\circ} \mathrm{C}$, coevaporated with toluene, and subjected to chromatography on silica gel (yields 70\%-90\%). Peracetylated derivatives of di- and trisialic acids $(\mathbf{1 9}+\mathbf{2 0}$ and $\mathbf{2 7 - 3 0})$ were mixtures of acetates of methyl ester and lactones, which were used without separation. 


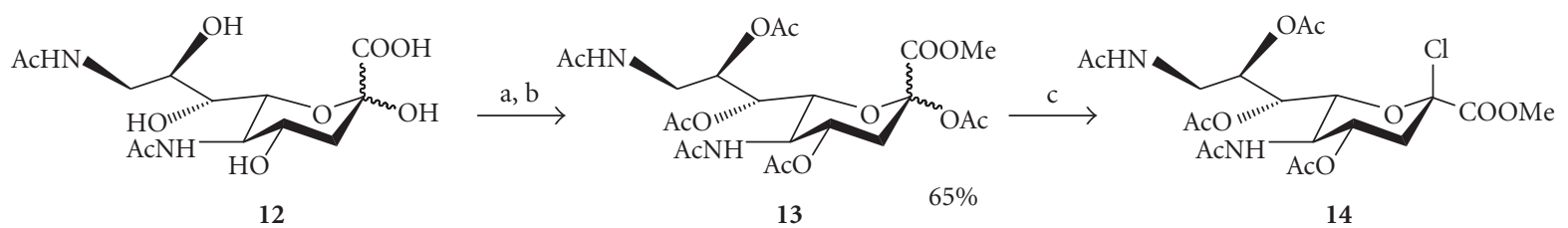

$14+\mathrm{HO}\left(\mathrm{CH}_{2}\right)_{3} \mathrm{NHCOCF}_{3} \stackrel{\mathrm{d}, \mathrm{e}, \mathrm{f}}{\longrightarrow}$

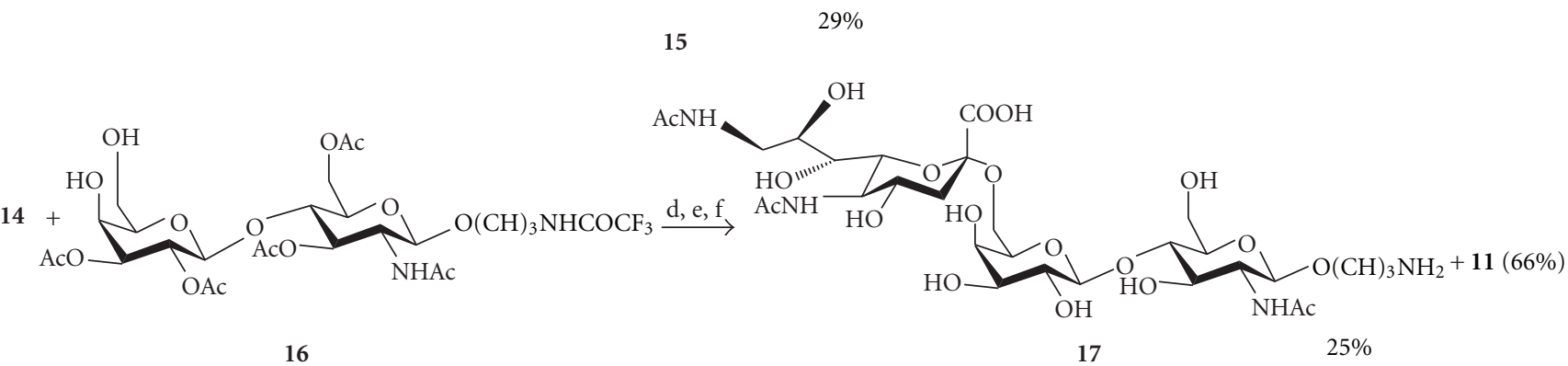

Scheme 2: (a) MeOH/HCl; (b) $\mathrm{Ac}_{2} \mathrm{O} / \mathrm{Py}$; (c) $\mathrm{AcCl}, \mathrm{MeOH} / \mathrm{CHCl}_{3}$; (d) $\mathrm{Ag}_{2} \mathrm{CO}_{3}, \mathrm{CH}_{2} \mathrm{Cl}_{2}, \mathrm{MS} 4 \AA$; (e) $\mathrm{MeONa} / \mathrm{MeOH}$; (f) $\mathrm{NaOH} / \mathrm{H}_{2} \mathrm{O}$.

TABLE 1: Sialylation with neuraminic acid derivatives.

\begin{tabular}{|c|c|c|c|c|c|c|c|c|c|c|}
\hline \multirow[b]{2}{*}{ \# } & \multirow[b]{2}{*}{ Sug-Cl } & \multirow[t]{2}{*}{$\underset{\substack{\text { protected } \\
\text { glycosylchloride }}}{\text { Sug-CI }}+\mathrm{ROH}$} & \multicolumn{2}{|c|}{$\underset{\text { or } \mathrm{Ag}_{2} \mathrm{CO}_{3}}{\stackrel{\mathrm{Ag}_{2} \mathrm{CO}_{3} / \mathrm{AgOTF}^{2}}{\longrightarrow}} \stackrel{\text { deprotec }}{ }$} & $\stackrel{\text { tion }}{\longrightarrow}$ separation & \multicolumn{2}{|l|}{$\rightarrow \underset{\substack{\text { unprotected } \\
\text { glycoside }}}{\text { Sug-OR }}$} & \multirow[b]{2}{*}{$\begin{array}{c}\text { Yield }^{\mathrm{a}} \\
\alpha+\beta, \%\end{array}$} & \multirow[b]{2}{*}{$\begin{array}{l}\text { Yield }^{\mathrm{a}} \\
\alpha, \%\end{array}$} & \multirow[b]{2}{*}{$\alpha / \beta^{\mathrm{b}}$} \\
\hline & & & $\begin{array}{l}\mathrm{ROH} / \\
\text { Sug-Cl }\end{array}$ & $\begin{array}{c}\mathrm{Ag}_{2} \mathrm{CO}_{3} / \\
\text { Sug-Cl }\end{array}$ & $\begin{array}{l}\text { AgOTf/ } \\
\text { Sug-Cl }\end{array}$ & $\begin{array}{l}\text { Time, } \\
\text { days }\end{array}$ & $\begin{array}{l}\text { Sug-OR } \\
\quad(\alpha)\end{array}$ & & & \\
\hline 1 & \multirow[t]{2}{*}{ Neu5Ac (3) } & $\mathrm{HO}\left(\mathrm{CH}_{2}\right)_{3} \mathrm{NHCOCF}_{3}$ & 5 & 2 & - & $2.6(62 \mathrm{~h})$ & \multirow[t]{2}{*}{4} & 71 & 71 & $\begin{aligned}> & 98: 2 \\
& {[8] }\end{aligned}$ \\
\hline $2^{c}$ & & $\mathrm{HO}\left(\mathrm{CH}_{2}\right)_{3} \mathrm{NHCOCF}_{3}$ & 2 & 3 & 0.1 & $0.2(5 \mathrm{~h})$ & & 47 & 36 & $95: 5$ \\
\hline 3 & \multirow{2}{*}{ Neu5Gc (7) } & $\mathrm{HO}\left(\mathrm{CH}_{2}\right)_{3} \mathrm{NHCOCF}_{3}$ & 3 & 3 & - & 3 & 8 & 68 & 57 & $92: 8$ \\
\hline 4 & & $\begin{array}{l}4^{\prime}, 6^{\prime}-\text { diol-LacNAc } \beta- \\
\mathrm{O}\left(\mathrm{CH}_{2}\right)_{3} \mathrm{NHCOCF}_{3}(9)^{\mathrm{d}}\end{array}$ & 1 & 3 & 0.1 & 7 & 10 & 30 & 12 & $43: 57$ \\
\hline 5 & \multirow[t]{2}{*}{$\begin{array}{l}\text { 9-deoxy-9-NAc- } \\
\text { Neu5Ac (14) }\end{array}$} & $\mathrm{HO}\left(\mathrm{CH}_{2}\right)_{3} \mathrm{NHCOCF}_{3}$ & 1 & 3 & - & 3 & 15 & n.d. & 29 & $\begin{array}{c}\text { Traces } \\
\text { of } \beta\end{array}$ \\
\hline 6 & & $\begin{array}{l}4^{\prime}, 6^{\prime}-\text { diol-LacNAc } \beta- \\
\mathrm{O}\left(\mathrm{CH}_{2}\right)_{3} \mathrm{NHCOCF}_{3}(\mathbf{1 6})^{\mathrm{e}}\end{array}$ & 1 & 6 & - & 5 & 17 & n.d. & 25 & $\begin{array}{c}\text { Traces } \\
\text { of } \beta\end{array}$ \\
\hline 7 & Neu5Ac2-8 & $\mathrm{HO}\left(\mathrm{CH}_{2}\right)_{3} \mathrm{NHCOCF}_{3}$ & 2 & 3 & - & 7 & \multirow[t]{2}{*}{23} & 20 & $\begin{array}{l}\text { not } \\
\text { done }\end{array}$ & $94: 6$ \\
\hline 8 & Neu5Ac 2-8 & $\mathrm{HO}\left(\mathrm{CH}_{2}\right)_{3} \mathrm{NHCOCF}_{3}$ & 2 & 3 & 0.1 & 1 & & 36 & 32 & $90: 10$ \\
\hline 9 & \multirow{3}{*}{$(21+22)$} & $\mathrm{MeOH}$ & 100 & 6 & 0.15 & 3 & 24 & n.d. & 40 & $\begin{array}{c}\text { Traces } \\
\text { of } \beta\end{array}$ \\
\hline 10 & & $\begin{array}{l}\mathrm{HOCH}_{2}(p- \\
\left.\mathrm{C}_{6} \mathrm{H}_{4}\right) \mathrm{NHCOCH}_{2} \mathrm{NHBoc}^{-}\end{array}$ & 2 & 5 & $0.1^{\mathrm{f}}$ & 3 & \multirow[t]{2}{*}{25} & 20 & 10 & $50: 50$ \\
\hline 11 & & $\begin{array}{l}\mathrm{HOCH}_{2}(p- \\
\left.\mathrm{C}_{6} \mathrm{H}_{4}\right) \mathrm{NHCOCH}_{2} \mathrm{NHBoc}^{-}\end{array}$ & 4 & 6 & 0.6 & 3 & & 32 & 12 & $39: 61$ \\
\hline 12 & $\begin{array}{l}\text { Neu5Ac2-8 } \\
\text { Neu5Ac2-8 }\end{array}$ & $\mathrm{HO}\left(\mathrm{CH}_{2}\right)_{3} \mathrm{NHCOCF}_{3}$ & 3 & 3 & $0.1^{\mathrm{f}}$ & 7 & \multirow[t]{2}{*}{32} & 33 & 27 & $81: 19$ \\
\hline 13 & Neu5Ac2-8 (31) & $\mathrm{HO}\left(\mathrm{CH}_{2}\right)_{3} \mathrm{NHCOCF}_{3}$ & 3 & 3 & 0.5 & 7 & & 37 & $\begin{array}{l}\text { not } \\
\text { done }\end{array}$ & $67: 33$ \\
\hline
\end{tabular}

a Preparative yield of unprotected glycoside Sug-OR calculated on protected precursor of Sug- $\mathrm{Cl}$ (n.d.: not determined);

$\mathrm{b} \alpha / \beta$ ratio according to NMR data;

${ }^{c} \mathrm{~A}$ series of experiments demonstrated that these conditions are optimal for the reaction in the presence of AgOTf;

${ }^{d}$ Recovery of the acceptor in the form $\mathrm{LacNAc} \beta-\mathrm{O}\left(\mathrm{CH}_{2}\right)_{3} \mathrm{NH}_{2}$ (11) $58 \%$;

e Recovery of the acceptor in the form $\mathrm{LacNAc} \beta-\mathrm{O}\left(\mathrm{CH}_{2}\right)_{3} \mathrm{NH}_{2}(\mathbf{1 1}) 66 \%$;

${ }_{\mathrm{f}}$ Reaction did not proceed without AgOTf. 


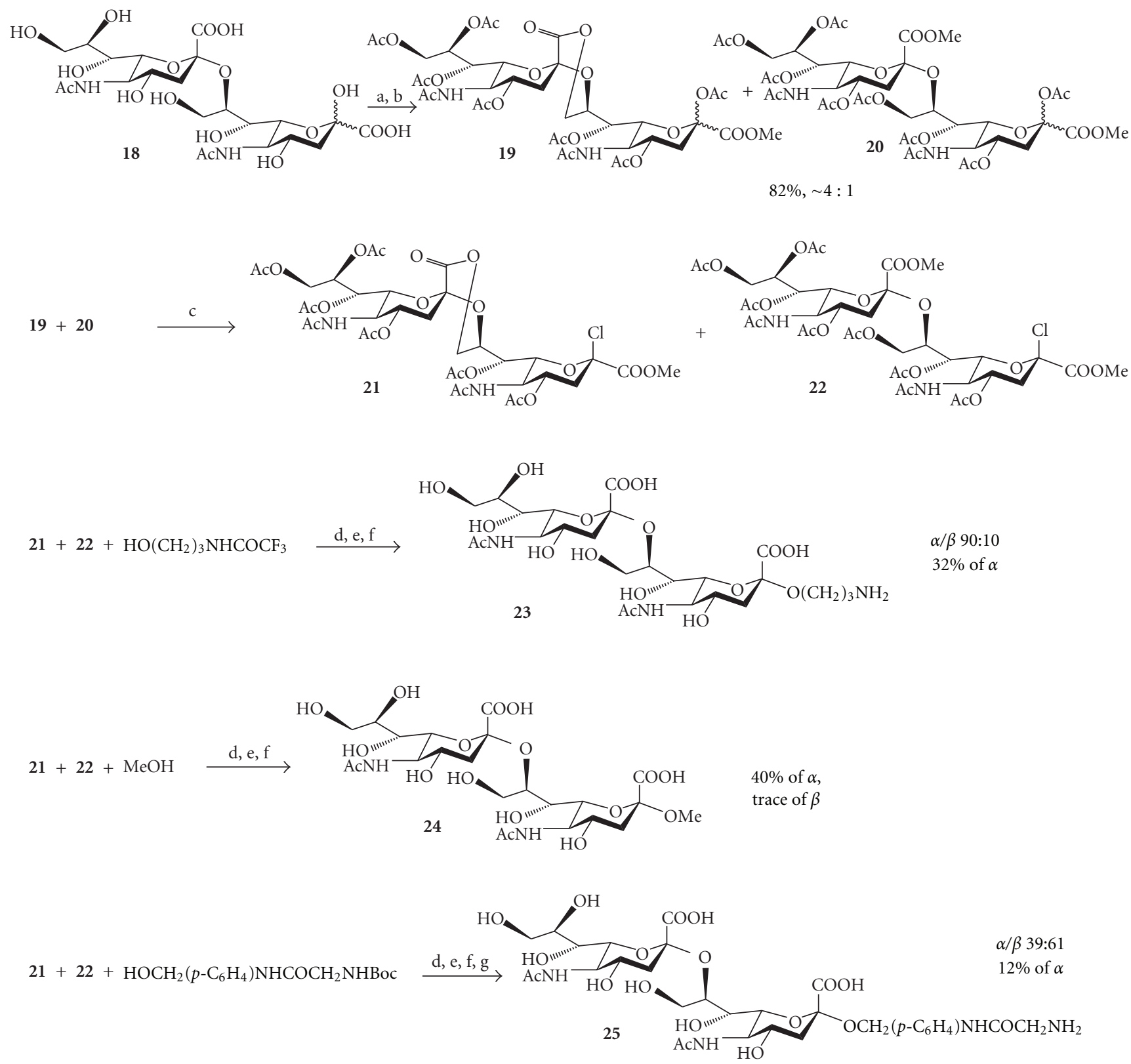

Scheme 3: (a) $\mathrm{MeOH} / \mathrm{HCl}$; (b) $\mathrm{Ac} 2 \mathrm{O} / \mathrm{Py}$; (c) $\mathrm{AcCl}, \mathrm{MeOH} / \mathrm{CHCl}_{3}$; (d) $\mathrm{Ag}_{2} \mathrm{CO}_{3} / \mathrm{AgOTf}, \mathrm{CH}_{2} \mathrm{Cl}_{2}, \mathrm{MS} 4 \AA$; (e) $\mathrm{MeONa} / \mathrm{MeOH}$; (f) $\mathrm{NaOH} / \mathrm{H}_{2} \mathrm{O}$; (g) $\mathrm{CF}_{3} \mathrm{COOH}$.

\subsection{Preparation of Neuraminic Acid Glycosyl Chlorides} Derivatives (Sug-Cl) General procedure for 7, 14, $21+22$, and 31. Peracetylated methyl ester of neuraminic acid derivative $(1 \mathrm{mmol}$ of $6,13,19+20$ or $27-30)$ was dissolved in $10 \mathrm{ml}$ freshly distilled chloroform followed by addition of dry methanol $(0.81 \mathrm{ml}, 20 \mathrm{mmol})$. The solution was cooled (ice + salt) and $\mathrm{AcCl}(2.84 \mathrm{ml}, 40 \mathrm{mmol})$ was added dropwise at cooling and stirring. After $30 \mathrm{~min}$ the reaction mixture was thoroughly sealed then warmed slowly and kept at room temperature for 1.5-2 days. The reaction mixture was evaporated at reduced pressure (water jet pump) and coevaporated with dry toluene several times to neutral $\mathrm{pH}$ value of the solution. Chlorides obtained in this way were used without further purification assuming quantitative yield of the reaction (the substances were stored at $-18^{\circ} \mathrm{C}$ ).

2.3. Glycosylation General procedure for $4,8,10,15,17$, 23, 24, 25, and 32 (see Table 1 for details). A solution of Sug- $\mathrm{Cl}(0.1 \mathrm{mmol})$ in dry $\mathrm{CH}_{2} \mathrm{Cl}_{2}(3 \mathrm{ml})$ and AgOTf (0.01-0.06 mmol) were added to a mixture of acceptor (0.1$0.4 \mathrm{mmol}), \mathrm{Ag}_{2} \mathrm{CO}_{3}(0.3-0.6 \mathrm{mmol})$, and MS $4 \AA(1 \mathrm{~g})$ in dry methylene chloride $(5 \mathrm{ml})$. The reaction mixture was stirred at room temperature $\left(20-25^{\circ} \mathrm{C}\right)$ for $0.2-7$ days (reaction completion was determined by disappearing of Sug-Cl according to TLC). The reaction mixture was filtered, the residue was washed with $\mathrm{CHCl}_{3} / \mathrm{MeOH}(1: 1)$; the combined 


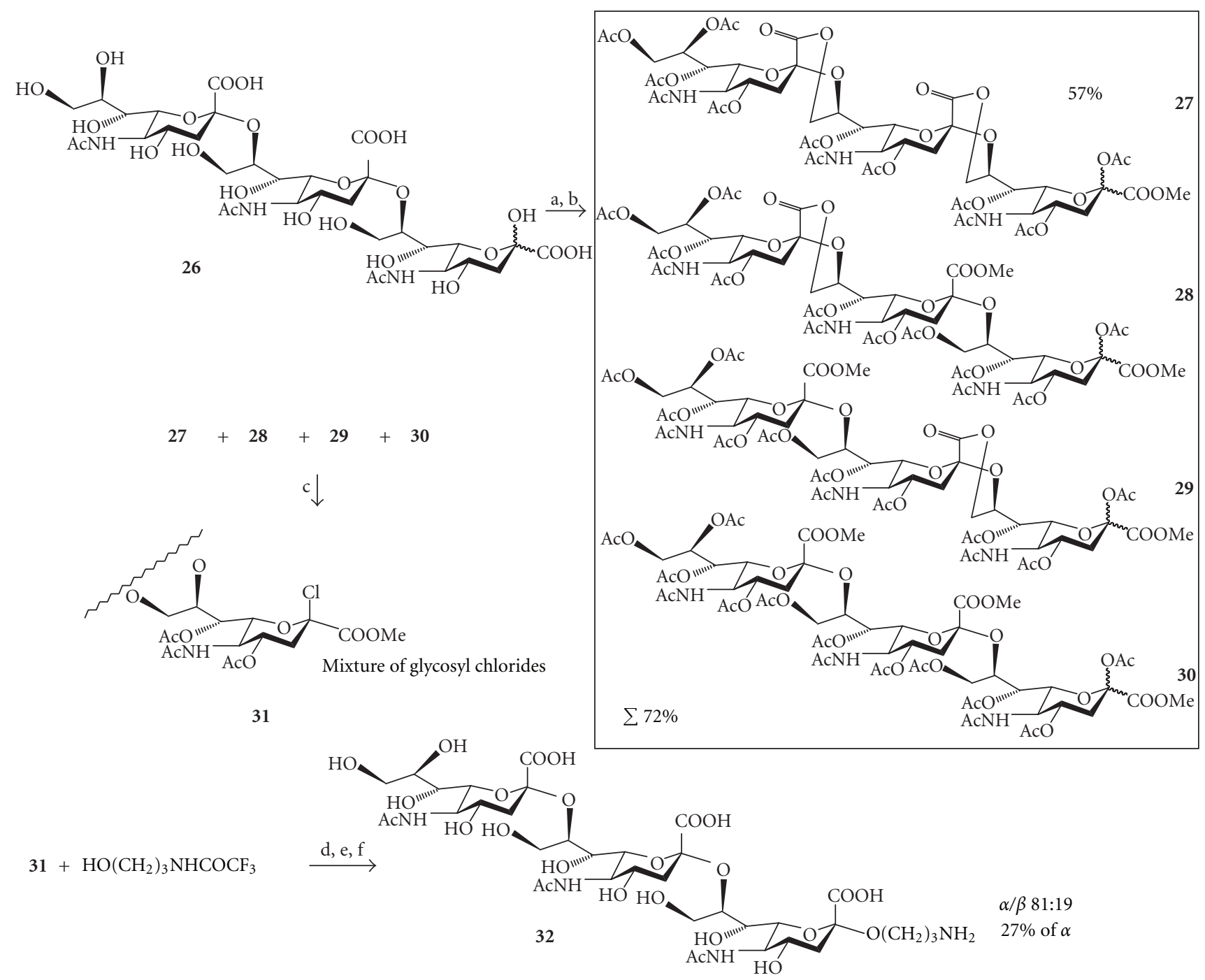

Scheme 4: (a) MeOH/HCl; (b) $\mathrm{Ac}_{2} \mathrm{O} / \mathrm{Py}$; (c) AcCl, $\mathrm{MeOH} / \mathrm{CHCl}_{3}$; (d) $\mathrm{Ag}_{2} \mathrm{CO}_{3} / \mathrm{AgOTf} \mathrm{CH}_{2} \mathrm{Cl}_{2}, \mathrm{MS} 4 \AA$; (e) $\mathrm{MeONa} / \mathrm{MeOH}$; (f) NaOH/H $2 \mathrm{O}$.

filtrate was evaporated, coevaporated with toluene, and dried in vacuo.

\subsection{Deprotection and Separation. (see Table 1 for yields).}

(a) Procedure for 4, 8, 15, and 17: the dry residue of treated reaction mixture (see above) was dissolved in $6 \mathrm{ml}$ of dry $\mathrm{MeOH}$ and $0.3 \mathrm{ml} 2 \mathrm{M} \mathrm{MeONa} / \mathrm{MeOH}$ were added. The mixture was kept for $30 \mathrm{~min}$ at r.t., then evaporated followed by addition of $6 \mathrm{ml} \mathrm{H}_{2} \mathrm{O}$. After 10-15 h (r.t.) the solution was evaporated, the residue was dissolved in $1 \mathrm{ml}$ water and the solution was applied on Dowex 50x4$400\left(\mathrm{H}^{+}\right)$ion-exchange resin column $(1.5 \times 6 \mathrm{~cm})$. The resin was washed sequentially with water $(50 \mathrm{ml}), 1 \mathrm{M}$ aq. pyridine $(50 \mathrm{ml})$ and $1 \mathrm{M}$ aq. $\mathrm{NH}_{3}(50 \mathrm{ml})$. Target glycosides were completely eluted with $1 \mathrm{M}$ pyridine. In the case of 10 and 17, nonreacted acceptor 16 was eluted with $1 \mathrm{M}$ aq. $\mathrm{NH}_{3}$ in deprotected form (11). Pure $\alpha$-glycoside 8 and corresponding $\beta$ anomer were obtained by HPLC separation (ODS $\mathrm{C}_{18}$, water). (b) Procedure for $\mathbf{1 0}$ was the same as (a), but included hydrogenolysis before $\mathrm{MeONa} / \mathrm{MeOH}$ treatment: $\mathrm{MeOH}$, $5 \% \mathrm{Pd} / \mathrm{C}, \mathrm{H}_{2}, 2 \mathrm{~h}$. Pure $\alpha$-glycoside 10 and corresponding $\beta$ anomer were obtained by HPLC separation (ODS $\mathrm{C}_{18}$, water).

(c) Synthesis of 23 and 32 included procedure (a); products were eluted $1 \mathrm{M}$ aq. pyridine. The obtained material was treated with $1 \mathrm{ml}$ of $0.1 \mathrm{M} \mathrm{NaOH}$ for $2 \mathrm{~h}$ at r.t. to open lactone rings (partial lactonisation occurs during purification on Dowex $\mathrm{H}^{+}$) followed by neutralization with AcOH. Pure products as $\mathrm{Na}$-salts were obtained after chromatography on silica gel $(\mathrm{MeOH} / \mathrm{MeCN} /$ water, 3:3:1), purification on Sephadex LH-20 (MeCN/water, 1:1), cation exchange on Dowex 50x4-400 $\left(\mathrm{Na}^{+}\right)$, and freeze drying.

(d) Procedure for 25 was the same as (c), with additional treatment with $\mathrm{CF}_{3} \mathrm{COOH}$ to remove Boc protection before the first Dowex chromatography. The mixture was dissolved in $0.3 \mathrm{ml} \mathrm{CF}_{3} \mathrm{COOH}$, kept for $1 \mathrm{~h}$ at r.t., then coevaporated with toluene and dried in vacuo; the followed procedures were performed according to procedure (c). 
(e) Synthesis of $\mathbf{2 4}$ included deprotection (treatment with $\mathrm{MeONa} / \mathrm{MeOH}, \mathrm{NaOH} / \mathrm{H}_{2} \mathrm{O}$, neutralization with $\mathrm{AcOH}$ ), column chromatography on silica gel (2propanol/MeCN/water $4: 3: 2$ ), purification on Sephadex LH-20 (MeCN/water, 1:1), cation exchange on Dowex 50x4$400\left(\mathrm{Na}^{+}\right)$and freeze drying.

2.4.1. Characteristics of Synthesized Spacered Saccharides. ${ }^{1} \mathrm{H}$ NMR spectra were recorded at $30^{\circ} \mathrm{C}$ with a Varian $600 \mathrm{MHz}$ instrument. Mass spectra (MALDI-TOF) were recorded on a Vision 2000 mass spectrometer. Optical rotations were measured with a JASCO DIP-360 polarimeter at $20^{\circ} \mathrm{C}$. Thinlayer chromatography was performed using silica gel 60 $\mathrm{F}_{254}$ aluminum sheets (Merck, 1.05554) with detection by charring after $7 \% \mathrm{H}_{3} \mathrm{PO}_{4}$ soaking, or ninhydrine.

9-Deoxy-9-NAc-Neu5Aco-O $\left(\mathrm{CH}_{2}\right)_{3} \mathrm{NH}_{2}$ (15). ${ }^{1} \mathrm{H} \quad \mathrm{NMR}$ spectrum $\left(\mathrm{D}_{2} \mathrm{O}, 303 \mathrm{~K}, 600 \mathrm{MHz}\right) \delta \mathrm{ppm}: 1.695(1 \mathrm{H}, \mathrm{dd}$, $\left.J_{3 \mathrm{ax}, 3 \mathrm{eq}} \approx J_{3 \mathrm{ax}, 4} \approx 12 \mathrm{~Hz}, \mathrm{H} 3 \mathrm{ax}\right), 1.953(2 \mathrm{H}, \mathrm{m}, 2 \mathrm{CH}), 2.035$ and $2.053\left(2 \times 3 \mathrm{H}, \mathrm{s}, 2 \mathrm{COCH}_{3}\right), 2.722\left(1 \mathrm{H}, \mathrm{dd}, J_{3 \mathrm{eq}, 3 \mathrm{ax}} 12,5\right.$, $\left.J_{3 \mathrm{eq}, 4} 4.7 \mathrm{~Hz}, \mathrm{H} 3 \mathrm{eq}\right), 3.138(2 \mathrm{H}, \mathrm{m}, 2 \mathrm{CHN}), 3.266(1 \mathrm{H}, \mathrm{dd}$, $\left.J_{9,9^{\prime}} 14.2, J_{9,8} 7.7 \mathrm{~Hz}, \mathrm{H} 9\right), 3.504\left(1 \mathrm{H}, \mathrm{dd}, J_{6,7} 1.4, J_{7,8} 8.8\right.$ $\mathrm{Hz}, \mathrm{H} 7), 3.584(1 \mathrm{H}, \mathrm{m}, \mathrm{OCH}), 3.629\left(1 \mathrm{H}, \mathrm{dd}, J_{9^{\prime}, 9} 14.2, J_{9^{\prime}, 8}\right.$ $\left.2.9 \mathrm{~Hz}, \mathrm{H}^{\prime}\right), 3.704\left(1 \mathrm{H}\right.$, ddd, $J_{4,3 \text { eq }} 4.7, J_{4,3 \text { ax }} 11.8, J_{4,5} 9.3 \mathrm{~Hz}$, H4), $3.788\left(1 \mathrm{H}, \mathrm{dd}, J_{6,5} 10.4, J_{6,7} 1.4 \mathrm{~Hz}, \mathrm{H} 6\right), 3.839(1 \mathrm{H}, \mathrm{dd}$, $\left.J_{5,6} 10.4, J_{5,4} 9.3 \mathrm{~Hz}, \mathrm{H} 5\right), 3.833(1 \mathrm{H}, \mathrm{m}, \mathrm{OCH}), 3.903(1 \mathrm{H}$, ddd, $\left.J_{8,9} 2.9, J_{8,9^{\prime}} 7.7, J_{8,7} 8.8 \mathrm{~Hz}, \mathrm{H} 8\right)$. MS, $\left.m / z: 408\left[\mathrm{M}^{-}\right]\right)$. $[\alpha]_{\mathrm{D}}+1.8\left(c 1, \mathrm{H}_{2} \mathrm{O}\right) . R_{f} 0.58(\mathrm{MeOH} / \mathrm{MeCN} /$ water $3: 3: 2)$.

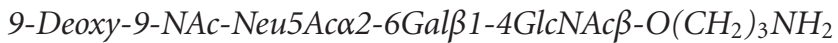
(inner salt) (17). ${ }^{1} \mathrm{H}$ NMR spectrum $\left(\mathrm{D}_{2} \mathrm{O}, \quad 303 \mathrm{~K}\right.$, $600 \mathrm{MHz}) \delta$ ppm: $1.716\left(1 \mathrm{H}, \mathrm{dd}, J_{3 \mathrm{ax}, 3 \mathrm{eq}} \approx J_{3 \mathrm{ax}, 4} \approx\right.$ $12.2 \mathrm{~Hz}, \mathrm{H} 3 \mathrm{ax}$ 9-deoxy-9-NAc-Neu5Ac), $1.975(2 \mathrm{H}, \mathrm{m}$, $2 \mathrm{CH}), 2.037,2.045,2.092\left(3 \times 3 \mathrm{H}, \mathrm{s}, 3 \mathrm{COCH}_{3}\right), 2.677(1 \mathrm{H}$, $\mathrm{dd}, J_{3 \text { eq } 4} 4.5, J_{3 \text { eq,3ax }} 12.2 \mathrm{~Hz}$, H3eq 9-deoxy-9-NAc-Neu5Ac), $3.114(2 \mathrm{H}, \mathrm{m}, 2 \mathrm{NCH}), 3.303\left(1 \mathrm{H}, \mathrm{dd}, J_{9,8} 7.7, J_{9,9^{\prime}} 14.0 \mathrm{~Hz}\right.$, H9 9-deoxy-9-NAc-Neu5Ac), $3.462\left(1 \mathrm{H}, \mathrm{dd}, J_{7,6} 1.4, J_{7,8}\right.$ 9.0 Hz, H7 9-deoxy-9-NAc-Neu5Ac), 3.560 (2H, m, H2, H6 Gal $\beta), 3.611\left(1 \mathrm{H}, \mathrm{dd}, J_{9^{\prime}, 8} 2.9, J_{9^{\prime}, .9} 14.0 \mathrm{~Hz}, \mathrm{H} 9^{\prime}\right.$ 9deoxy-9-NAc-Neu5Ac), 3.61-3.71 (4H, m, H4, H5 GlcNAc $\beta$, $J_{3,4} 3.5, J_{3,2} 9.8 \mathrm{~Hz}, \mathrm{H} 3 \mathrm{Gal} \beta, \mathrm{H} 4$ 9-deoxy-9-NAc-Neu5Ac), 3.71-3.88 (7H, m, H2, H3 and H6 GlcNAc $\beta, \mathrm{H} 5 \mathrm{Gal} \beta, \mathrm{H} 5$, H6 9-deoxy-9-NAc-Neu5Ac, OCH), 3.945 (2H, m, H4 Gal $\beta$, H8 9-deoxy-9-NAc-Neu5Ac), 3.97-4.08, (3H, m, OCH, H6' of GlcNAc $\beta, \mathrm{H}^{\prime}$ of $\left.\mathrm{Gal} \beta\right), 4.464\left(1 \mathrm{H}, \mathrm{d}, J_{1,2} 7.9 \mathrm{~Hz}, \mathrm{H} 1\right.$ $\mathrm{Gal} \beta), 4.560(1 \mathrm{H}, \mathrm{m}, \mathrm{H1} \operatorname{GlcNAc} \beta)$. MS, $m / z: 771\left[\mathrm{M}^{-}\right]$. $[\alpha]_{\mathrm{D}}-20\left(c 1, \mathrm{H}_{2} \mathrm{O}\right) . R_{f} 0.41(\mathrm{MeOH} / \mathrm{MeCN} /$ water $3: 3: 2)$.

$\mathrm{Neu} 5 \mathrm{Ac \alpha} 2-8 \mathrm{Neu} 5 \mathrm{Ac \alpha}-\mathrm{O}\left(\mathrm{CH}_{2}\right)_{3} \mathrm{NH}_{2} \quad\left(\mathrm{Na}^{+}\right.$salt $) \quad(23) .{ }^{1} \mathrm{H}$ NMR spectrum $\left(\mathrm{D}_{2} \mathrm{O}, 303 \mathrm{~K}, 600 \mathrm{MHz}\right) \delta \mathrm{ppm}: 1.646(1 \mathrm{H}$, $\left.\mathrm{dd}, \mathrm{J}_{3 \mathrm{ax}, 3 \mathrm{eq}} \approx J_{3 \mathrm{ax}, 4} \approx 12 \mathrm{~Hz}, \mathrm{H} 3 \mathrm{ax} \operatorname{Neu} 5 \mathrm{Ac} \alpha\right), 1.751(1 \mathrm{H}, \mathrm{dd}$, $\mathrm{J}_{3 \mathrm{ax}, 3 \mathrm{eq}} \approx J_{3 \mathrm{ax}, 4} \approx 12 \mathrm{~Hz}, \mathrm{H} 3 \mathrm{ax}$ Neu5Ac $\left.\alpha 2-8\right), 1.963(2 \mathrm{H}, \mathrm{m}$, $2 \mathrm{CH}), 2.050$ and $2.087\left(2 \times 3 \mathrm{H}, \mathrm{s}, 2 \mathrm{COCH}_{3}\right), 2.623(1 \mathrm{H}$, dd, $J_{3 \text { eq,3ax }} 12.4, J_{3 \text { eq, } 4} 4.4 \mathrm{~Hz}, \mathrm{H} 3$ eq Neu5Ac $\left.\alpha\right), 2.790(1 \mathrm{H}$, dd, $J_{3 \text { eq,3ax }} 12.4, J_{3 \text { eq, } 4} 4.5 \mathrm{~Hz}$, H3eq Neu5Ac $\left.2-8\right), 3.148(2 \mathrm{H}$, m, 2CHN), 3.55-3.76 (7H, m, OCH, H4, H9 Neu5Ac $\alpha, \mathrm{H} 4$, H6, H7, H9 Neu5Aca2-8), 3.79-3.94 (7H, m, OCH, H5, H6, H7 Neu5Ac $\alpha$, H5, H8, H9' Neu5Ac $\alpha 2-8), 4.113(1 \mathrm{H}$, dd. $J_{9^{\prime}, 8} 3.9, J_{9^{\prime}, 9} 12.1 \mathrm{~Hz}, \mathrm{H} 9^{\prime}$ Neu5Ac $\left.\alpha\right), 4.198(1 \mathrm{H}, \mathrm{m}, \mathrm{H} 8$ Neu5Ac $\alpha)$. MS, $m / z: 723\left[\mathrm{M}^{-}\right] .[\alpha]_{\mathrm{D}}+3.2\left(c 0.5, \mathrm{H}_{2} \mathrm{O}\right) . R_{f}$ $0,44(\mathrm{MeOH} / \mathrm{MeCN} /$ water $3: 3: 1)$.

$\mathrm{Neu} 5 \mathrm{Ac} \alpha 2-8 \mathrm{Neu} 5 \mathrm{Ac} \alpha-\mathrm{OCH}_{2}\left(\mathrm{p}-\mathrm{C}_{6} \mathrm{H}_{4}\right) \mathrm{NHCOCH}_{2} \mathrm{NH}_{2}\left(\mathrm{Na}^{+}\right.$ salt) (25). ${ }^{1} \mathrm{H}$ NMR spectrum $\left(\mathrm{D}_{2} \mathrm{O}, 303 \mathrm{~K}, 600 \mathrm{MHz}\right) \delta$ ppm: $1.651\left(1 \mathrm{H}, \mathrm{dd}, J_{3 \mathrm{ax}, 3 \mathrm{eq}} \approx J_{3 \mathrm{ax}, 4} \approx 12 \mathrm{~Hz}, \mathrm{H} 3 \mathrm{ax}\right.$ Neu5Ac $\alpha), 1.760\left(1 \mathrm{H}, \mathrm{dd}, \mathrm{J}_{3 \mathrm{ax}, 3 \mathrm{eq}} \approx J_{3 \mathrm{ax}, 4} \approx 12 \mathrm{~Hz}, \mathrm{H} 3 \mathrm{ax}\right.$ Neu5Ac $22-8), 2.051$ and $2.094\left(2 \times 3 \mathrm{H}, \mathrm{s}, 2 \mathrm{COCH}_{3}\right), 2.705$ $\left(1 \mathrm{H}, \mathrm{dd}, J_{3 \text { eq }, 3 \mathrm{ax}} 12.2, J_{3 \text { eq, } 4} 4.3 \mathrm{~Hz}, \mathrm{H} 3\right.$ eq Neu $\left.5 \mathrm{Ac} \alpha\right), 2.793$ $\left(1 \mathrm{H}, \mathrm{dd}, J_{3 \mathrm{eq}, 3 \mathrm{ax}} 12.4, J_{3 \mathrm{eq}, 4} 4.6 \mathrm{~Hz}, \mathrm{H} 3 \mathrm{eq}\right.$ Neu5Ac $\left.\alpha 2-8\right), 3.58-$ $3.75(6 \mathrm{H}, \mathrm{m}, \mathrm{H} 4, \mathrm{H} 9 \mathrm{Neu} 5 \mathrm{Ac} \alpha, \mathrm{H} 4, \mathrm{H} 6, \mathrm{H} 7, \mathrm{H} 9 \mathrm{Neu} 5 \mathrm{Ac} \alpha 2-$ 8), 3.81-3.95 (6H, m, H5, H6, H7 Neu5Ac $\alpha, \mathrm{H} 5, \mathrm{H} 8, \mathrm{H} 9$ ' Neu5Ac $\alpha 2-8), 3.954(2 \mathrm{H}, \mathrm{s}, 2 \mathrm{CHN}), 4.188\left(1 \mathrm{H}, \mathrm{dd}, J_{9^{\prime}, 9} 12.1\right.$, $J_{9^{\prime}, 8}$ 3.8, H9' Neu5Ac $\left.\alpha\right), 4.247$ (1H, m, H8 Neu5Ac $\alpha$ ), 4.504 and $4.504\left(2 \times 1 \mathrm{H}, \mathrm{d}, J_{\text {hem }} 10.8 \mathrm{~Hz}, 2 \mathrm{OCH}-\mathrm{Ar}\right), 7.478(4 \mathrm{H}$, m, $\left.\mathrm{C}_{6} \mathrm{H}_{4}\right)$. MS, $\left.m / z: 763\left[\mathrm{M}^{-}\right]\right) .[\alpha]_{\mathrm{D}}-17.0\left(c 0.2, \mathrm{H}_{2} \mathrm{O}\right) . R_{f}$ $0.60(\mathrm{MeOH} / \mathrm{MeCN} /$ water $3: 3: 2)$.

$\mathrm{Neu} 5 \mathrm{Ac} \alpha 2-8 \mathrm{Neu} 5 \mathrm{Ac} \beta-\mathrm{OCH}_{2}\left(\mathrm{p}-\mathrm{C}_{6} \mathrm{H}_{4}\right) \mathrm{NHCOCH}_{2} \mathrm{NH}_{2} \quad\left(\mathrm{Na}^{+}\right.$ salt) $(25 \beta) .{ }^{1} \mathrm{H}$ NMR spectrum $\left(\mathrm{D}_{2} \mathrm{O}, 303 \mathrm{~K}, 600 \mathrm{MHz}\right)$ $\delta$ ppm: $1.620\left(1 \mathrm{H}, \mathrm{dd}, \mathrm{J}_{3 \mathrm{ax}, 3 \mathrm{eq}} \approx J_{3 \mathrm{ax}, 4} \approx 12 \mathrm{~Hz}, \mathrm{H} 3 \mathrm{ax}\right.$ Neu5Ac $22-8), 1.690\left(1 \mathrm{H}\right.$, dd, $\mathrm{J}_{3 \mathrm{ax}, 3 \mathrm{eq}} \approx J_{3 \mathrm{ax}, 4}, \mathrm{H} 3 \mathrm{ax}$ Neu5Ac $\beta), 2.035$ and $2.105\left(2 \times 3 \mathrm{H}, \mathrm{s}, 2 \mathrm{COCH}_{3}\right), 2.391(1 \mathrm{H}$, dd, $J_{3 e q, 3 a x} 13.2, J_{3 e q, 4} 4.8 \mathrm{~Hz}, \mathrm{H} 3$ eq Neu5Ac $\left.\beta\right), 2.630(1 \mathrm{H}, \mathrm{dd}$, $J_{3 \text { eq,3ax }} 12.4, J_{3 \text { eq, } 4} 4.7 \mathrm{~Hz}, \mathrm{H} 3$ eq Neu5Ac $\left.\alpha 2-8\right), 3.52-3.64(4 \mathrm{H}$, m, H4, H6, H7, H9 Neu5Ac $22-8), 3.685\left(1 \mathrm{H}, \mathrm{dd}, J_{9^{\prime}, 9} 12.1\right.$, $J_{9^{\prime}, 8}, \mathrm{H}^{\prime}$ Neu5Ac $\left.\alpha 2-8\right), 3.71-3.81(4 \mathrm{H}, \mathrm{m}, \mathrm{H} 5$ Neu5Ac $\alpha 2-$ 8, H6, H7, H9 Neu5Ac $\beta$ ), 3.87-3.94 (2H, m, H5 Neu5Ac $\beta$, H8 Neu5Ac $\alpha 2-8), 3.955(2 \mathrm{H}, \mathrm{s}, 2 \mathrm{CHN}), 4.083(1 \mathrm{H}$, ddd, H4 Neu5Ac $\beta$ ), 4.158 ( $\left.2 \mathrm{H}, \mathrm{m}, \mathrm{H} 8, \mathrm{H} 9^{\prime} \mathrm{Neu} 5 \mathrm{Ac} \beta\right), 4.355$ and $4.542\left(2 \times 1 \mathrm{H}, 2 \mathrm{~d}, J_{\text {hem }} 10.8 \mathrm{~Hz}, 2 \mathrm{OCH}-\mathrm{Ar}\right), 7.527(4 \mathrm{H}, \mathrm{m}$, $\left.\mathrm{C}_{6} \mathrm{H}_{4}\right)$. MS, $\left.m / z: 763\left[\mathrm{M}^{-}\right]\right) .[\alpha]_{\mathrm{D}}+10.0\left(c 0.2, \mathrm{H}_{2} \mathrm{O}\right) \cdot R_{f} 0.40$ $(\mathrm{MeOH} / \mathrm{MeCN} /$ water $3: 3: 2)$.

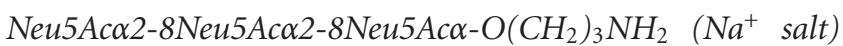
(32). ${ }^{1} \mathrm{H}$ NMR spectrum $\left(\mathrm{D}_{2} \mathrm{O}, 303 \mathrm{~K}, 600 \mathrm{MHz}\right) \delta$ ppm: $1.652,1.724$ and $1.767\left(3 \times 1 \mathrm{H}, \mathrm{dd}, J_{3 \mathrm{ax}, 3 \mathrm{eq}} \approx J_{3 \mathrm{ax}, 4}, 3 \times\right.$ H3ax), $1.965(2 \mathrm{H}, \mathrm{m}, 2 \mathrm{CH}), 2.051,2.089$ and $2.097(3 \times$ $\left.3 \mathrm{H}, \mathrm{s}, 3 \mathrm{COCH}_{3}\right), 2.621\left(1 \mathrm{H}, \mathrm{dd}, J_{3 \text { eq, 3ax }} 4.7, J_{3 \text { eq, } 4} 12.2 \mathrm{~Hz}\right.$, H3eq Neu5Ac $\alpha), 2.701\left(1 \mathrm{H}\right.$, dd, $J_{3 e q, 3 a x} 12.0, J_{3 e q, 4} 4.3 \mathrm{~Hz}$, H3eq 2-8Neu5Aca2-8), $2.784\left(1 \mathrm{H}\right.$, dd, $J_{3 e q, 3 a x} 4.7, J_{3 e q, 4}$ $12.2 \mathrm{~Hz}, \mathrm{H} 3$ eq Neu5Ac $2-8), 3.150(2 \mathrm{H}, \mathrm{m}, 2 \mathrm{CHN}), 3.55-$ $3.74(10 \mathrm{H}, \mathrm{m}, \mathrm{OCH}, 3 \times \mathrm{H} 4,3 \times \mathrm{H} 9$; H6, H7 Neu5Aca28, H6 2-8Neu5Ac 2 2-8), 3.79-3.97 (9H, m, 3× H5; H6, H7 Neu5Ac $\alpha$, H7 2-8Neu5Ac $\alpha 2-8$, H8, H9' Neu5Ac 2 2-8, $\mathrm{OCH}), 4.112\left(3 \mathrm{H}, \mathrm{m}, \mathrm{H} 8 \mathrm{Neu} 5 \mathrm{Ac} \alpha, \mathrm{H} 8, \mathrm{H}^{\prime}\right.$ 2-8Neu5Ac $\alpha 2-$ 8). MS, $\left.m / z: 949\left[\mathrm{M}^{-}\right]\right) \cdot[\alpha]_{\mathrm{D}}+9.2\left(c 1, \mathrm{H}_{2} \mathrm{O}\right) . R_{f} 0.33$ $(\mathrm{MeOH} / \mathrm{MeCN} /$ water $3: 3: 1)$.

Neu5Aca2-8Neu5Aca2-8Neu5Ac $\beta-O\left(\mathrm{CH}_{2}\right)_{3} \mathrm{NH}_{2} \quad\left(\mathrm{Na}^{+}\right.$salt $)$ (32ß). ${ }^{1} \mathrm{H}$ NMR spectrum $\left(\mathrm{D}_{2} \mathrm{O}, 303 \mathrm{~K}, 600 \mathrm{MHz}\right) \delta \mathrm{ppm}$ : $1.653,1.674$ and $1.739\left(3 \times 1 \mathrm{H}, \mathrm{dd}, \mathrm{H}_{\mathrm{ax}}\right), 2.015(2 \mathrm{H}, \mathrm{m}$, $2 \mathrm{CH}), 2.050,2.086$ and $2.112\left(3 \times 3 \mathrm{H}, \mathrm{s}, 3 \mathrm{COCH}_{3}\right), 2.328$ $\left(1 \mathrm{H}, \mathrm{dd}, J_{3 \text { eq,3ax }} 12.9, J_{3 \text { eq, } 4} 4.9 \mathrm{~Hz}, \mathrm{H} 3\right.$ eq Neu $\left.5 \mathrm{Ac} \beta\right), 2.730$ $\left(1 \mathrm{H}, \mathrm{dd}, J_{3 \text { eq,3ax }} 12.4, J_{3 \text { eq, } 4} 4.2 \mathrm{~Hz}\right.$, H3eq 2-8Neu5Ac $\left.\alpha 2-8\right)$, $2.775\left(1 \mathrm{H}\right.$, dd, $J_{3 \text { eq, 3ax }} 12.3, J_{3 \text { eq, } 4} 4.5 \mathrm{~Hz}, \mathrm{H} 3$ eq Neu5Ac $\alpha 2-$ 8), $3.130(1 \mathrm{H}, \mathrm{m}, \mathrm{NCH}), 3.236(1 \mathrm{H}, \mathrm{m}, \mathrm{NCH}), 3.386$ 
(1H, m, OCH), 3.78-3.93 (6H, m, 3× H5; H7 Neu5Ac $\beta$, H7 2-8Neu5Ac $\alpha 2-8, \mathrm{H}^{\prime}$ Neu5Ac $\left.\alpha 2-8\right), 3.955$ (1H, m, H8 Neu5Ac $\alpha 2-8), 4.009$ (1H, ddd, $J_{4^{\prime}, 3 \text { eq }} 4.8, J_{4,3 \mathrm{ax}} \approx J_{4,5} \approx$ $11.0 \mathrm{~Hz}, \mathrm{H} 4 \mathrm{Neu} 5 \mathrm{Ac} \beta), 4.064\left(1 \mathrm{H}, \mathrm{dd}, J_{9^{\prime}, 9} 12.3, J_{9^{\prime}, 8} 2.6 \mathrm{~Hz}\right.$, H9' Neu5Ac $\beta$ ), 4.096 (1H, m, H8 Neu5Ac $\beta$ ), 4.159 (2H, m, H8, H9' 2-8Neu5Ac $\alpha 2-8)$. MS, $\left.m / z: 949\left[\mathrm{M}^{-}\right]\right)$. $[\alpha]_{\mathrm{D}}+9.0(c$ $\left.0.3, \mathrm{H}_{2} \mathrm{O}\right) . R_{f} 0.22(\mathrm{MeOH} / \mathrm{MeCN} /$ water $3: 3: 1)$.

\section{Acknowledgments}

This work was supported with FP6 (project "Avian influenza: impact of virus-host interactions on pathogenesis and ecology", acronym: FLUPATH, contract No. 044220) and Grant of Russian Foundation for Basic Research 07-04-00630.

\section{References}

[1] R. Schauer, "Achievements and challenges of sialic acid research," Glycoconjugate Journal, vol. 17, no. 7-9, pp. 485-499, 2000.

[2] A. Varki, "Sialic acids in human health and disease," Trends in Molecular Medicine, vol. 14, no. 8, pp. 351-360, 2008.

[3] G.-J. Boons and A. V. Demchenko, "Recent advances in $O$ sialylation," Chemical Reviews, vol. 100, no. 12, pp. 4539-4565, 2000.

[4] R. L. Halcomb and M. D. Chappell, "Recent developments in technology for glycosylation with sialic acid," Journal of Carbohydrate Chemistry, vol. 21, no. 7-9, pp. 723-768, 2002.

[5] Y. Liu, X. Ruan, X. Li, and Y. Li, "Efficient synthesis of a sialic acid $\alpha(2 \rightarrow 3)$ galactose building block and its application to the synthesis of ganglioside GM3," Journal of Organic Chemistry, vol. 73, no. 11, pp. 4287-4290, 2008.

[6] G. Pazynina, A. Tuzikov, A. Chinarev, P. Obukhova, and N. Bovin, "Simple stereoselective synthesis of $\alpha 2-6$ sialooligosaccharides," Tetrahedron Letters, vol. 43, no. 45, pp. 8011-8013, 2002.

[7] T. Yamaji, T. Teranishi, M. S. Alphey, P. R. Crocker, and Y. Hashimoto, "A small region of the natural killer cell receptor, Siglec-7, is responsible for its preferred binding to $\alpha 2,8$-disialyl and branched $\alpha 2,6$-sialyl residues. A comparison with Siglec9," Journal of Biological Chemistry, vol. 277, no. 8, pp. 63246332, 2002.

[8] L. A. Simeoni, A. B. Tuzikov, N. E. Byramova, and N. V. Bovin, "Neu5Ac and Neu5Gc ethylthioglycoside derivatives as glycosyl donors. Synthesis of aminopropyl glycosides of $\mathrm{N}$-acetyl and N-glycolylneuraminic acid," Russian Journal of Bioorganic Chemistry, vol. 23, pp. 139-146, 1997.

[9] A. Hasegawa, H. Ishida, and M. Kiso, "Synthetic studies on sialoglycoconjugates 46: a facile total synthesis of ganglioside $\mathrm{GD}_{3}$," Journal of Carbohydrate Chemistry, vol. 12, pp. 371-376, 1993.

[10] H. Ando, H. Ishida, M. Kiso, and A. Hasegawa, "A synthetic approach to the c-series gangliosides containing sialyl- $\alpha(2 \rightarrow$ $8)$ sialyl- $\alpha(2 \rightarrow$ and $)$ sialic acid: synthesis of ganglioside GT4, $\alpha(2 \rightarrow 6)$ GT4 and GT3," Carbohydrate Research, vol. 300, no. 3, pp. 207-217, 1997.

[11] A. B. Tuzikov, A. S. Gambaryan, L. R. Juneja, and N. V. Bovin, "Conversion of complex sialooligosaccharides into polymeric conjugates and their anti-influenza virus inhibitory potency," Journal of Carbohydrate Chemistry, vol. 19, no. 9, pp. 11911200, 2000.
[12] S. Z. Abbas, S. Sugiyama, J. Diakur, R. A. Pon, and R. Roy, "Synthesis of $\alpha$ - and $\beta$-methyl Neu5Ac $\alpha$ - $(2 \rightarrow 8)$ Neu5Ac disaccharides," Journal of Carbohydrate Chemistry, vol. 9, no. 6, pp. 891-901, 1990.

[13] A. A. Sherman, O. N. Yudina, A. S. Shashkov, V. M. Menshov, and N. E. Nifant'ev, "Synthesis of Neu5Ac- and Neu5Gc- $\alpha$ - $\left(2 \rightarrow 6^{\prime}\right)$-lactosamine 3-aminopropyl glycosides," Carbohydrate Research, vol. 330, no. 4, pp. 445-458, 2001.

[14] H. J. Gross, A. Bünsch, J. C. Paulson, and R. Brossmer, "Activation and transfer of novel synthetic 9-substituted sialic acids," European Journal of Biochemistry, vol. 168, no. 3, pp. 595-602, 1987.

[15] F. Michon, J.-R. Brisson, and H. J. Jennings, "Conformational differences between linear $\alpha(2 \rightarrow$ 8)-linked homosialooligosaccharides and the epitope of the group $B$ meningococcal polysaccharide," Biochemistry, vol. 26, no. 25, pp. 8399-8405, 1987.

[16] E. M. Rapoport, G. V. Pazynina, M. A. Sablina, P. R. Crocker, and N. V. Bovin, "Probing sialic acid binding Ig-like lectins (siglecs) with sulfated oligosaccharides," Biochemistry, vol. 71, no. 5, pp. 496-504, 2006.

[17] O. A. Kost, N. V. Bovin, E. E. Chemodanova, V. V. Nasonov, and T. A. Orth, "New feature of angiotensin-converting enzyme: carbohydrate-recognizing domain," Journal of Molecular Recognition, vol. 13, no. 6, pp. 360-369, 2000.

[18] E. M. Rapoport, YU. B. Sapot'ko, G. V. Pazynina, V. K. Bojenko, and N. V. Bovin, "Sialoside-binding macrophage lectins in phagocytosis of apoptotic bodies," Biochemistry, vol. 70, no. 3, pp. 330-338, 2005.

[19] R. Brossmer and H. J. Gross, "Sialic acid analogs and application for preparation of neoglycoconjugates," Methods in Enzymology, vol. 247, pp. 153-176, 1994. 


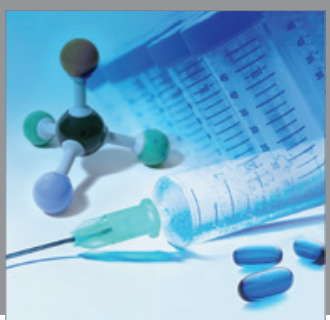

International Journal of

Medicinal Chemistry

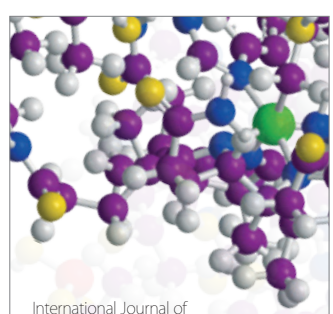

Carbohydrate Chemistry

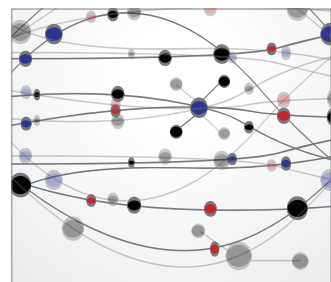

The Scientific World Journal
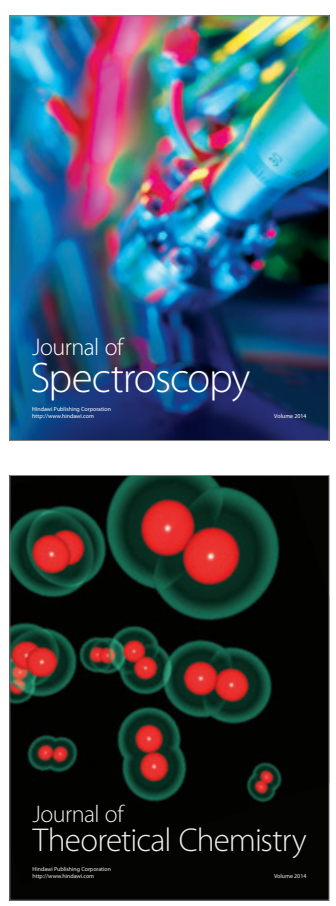
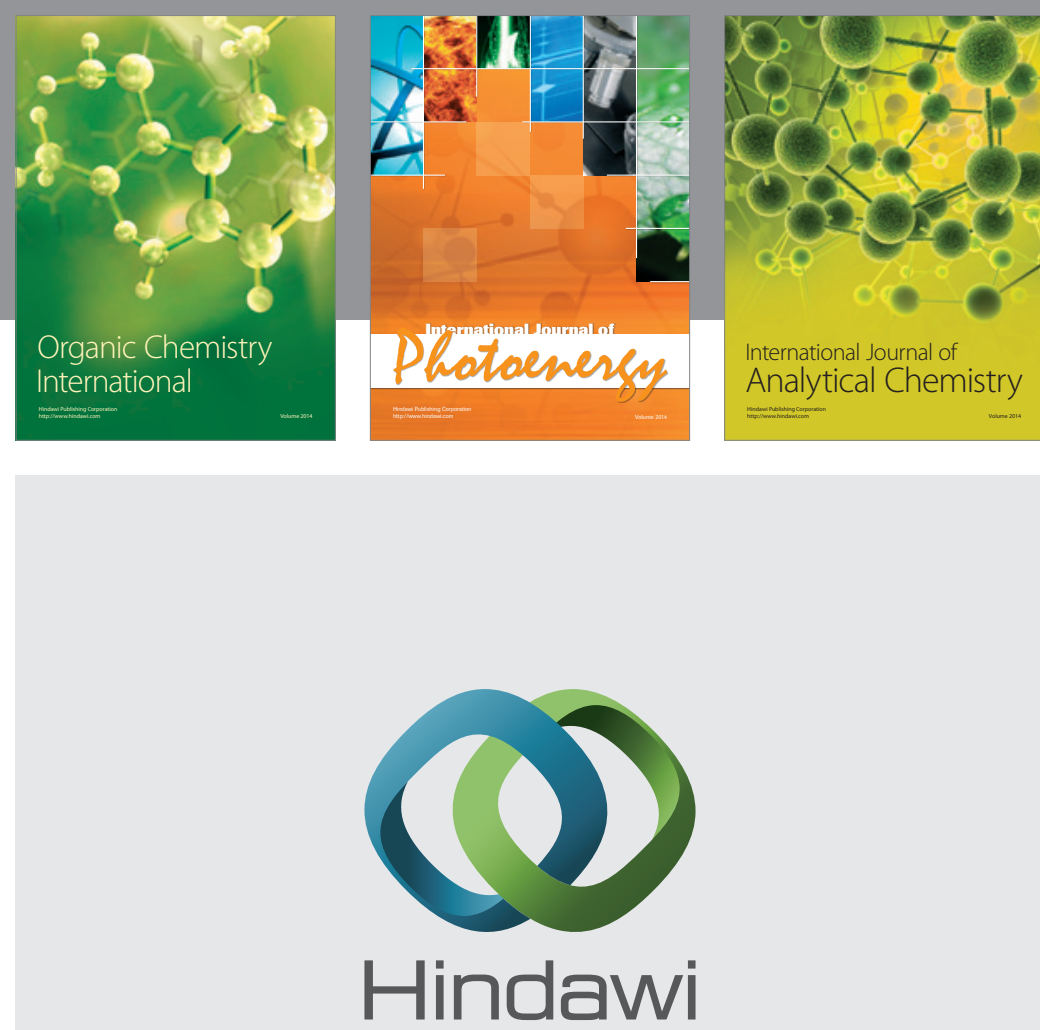

Submit your manuscripts at

http://www.hindawi.com
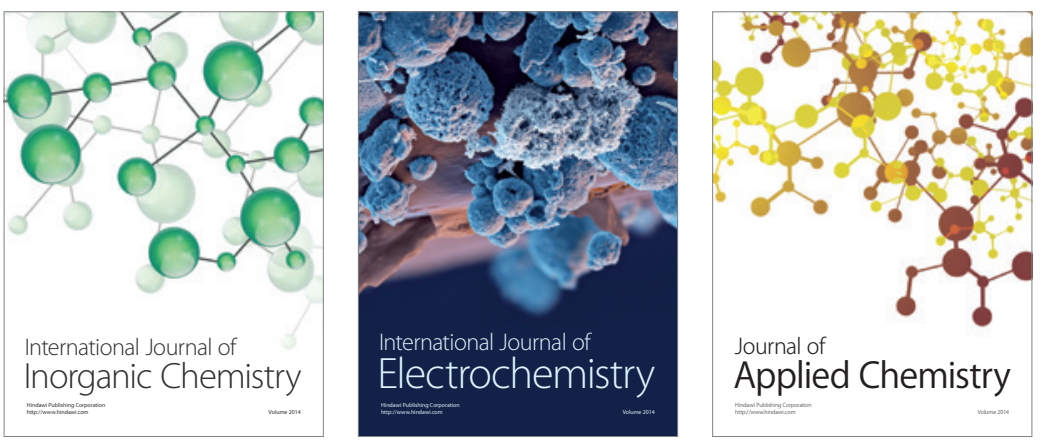

Journal of

Applied Chemistry
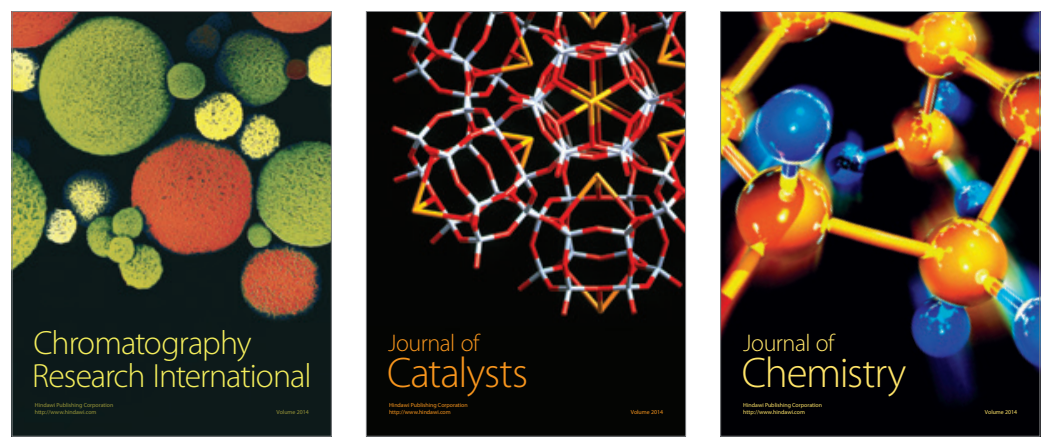
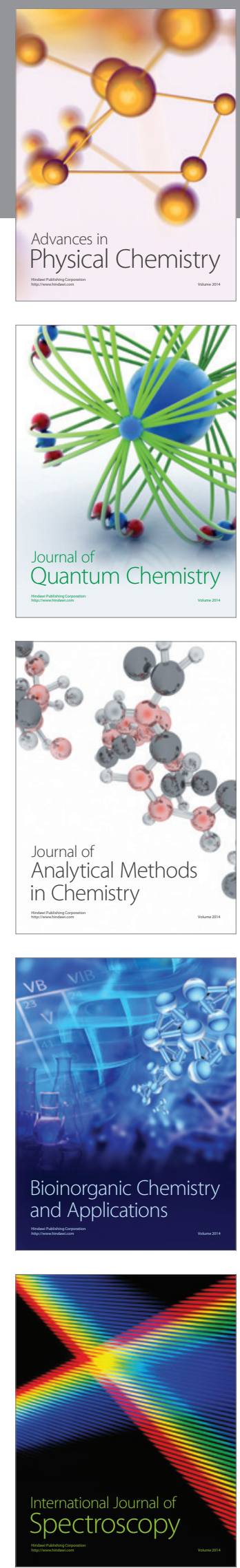